\title{
Economics
}

The Open-Access, Open-Assessment E-Journal

Vol. 12, 2018-57 | September 12, 2018 | http://dx.doi.org/10.5018/economics-ejournal.ja.2018-57

\section{Social limits to redistribution and conspicuous norms}

\section{Luca Ferrari}

\begin{abstract}
In this article the author considers an economy in which individuals are matched into pairs and the desirability of an individual depends on her position on the distribution of wealth. He assumes that individuals show their relative standing by consuming a conspicuous good and he shows that there exist different social norms supporting different matching arrangements. In addition, individuals have to vote over a redistributive policy and the author shows that, despite the desirability of the full redistributive outcomes, under some economic conditions the medium class is able to match with the high class in exchange of a minimum level of redistribution of wealth which keeps the low class far from economic and social opportunities.
\end{abstract}

(Published in Special Issue The economics of social status)

JEL D11 D31 D62

Keywords Social status; conspicuous consumption; matching; redistribution of wealth; income inequality; social organizations

\section{Authors}

Luca Ferrari, University of Corsica, Corte, France, luca.ferrari.sdc@gmail.com

Citation Luca Ferrari (2018). Social limits to redistribution and conspicuous norms. Economics: The Open-Access, Open-Assessment E-Journal, 12 (2018-57): 1-21. http://dx.doi.org/10.5018/economics-ejournal.ja.2018-57 


\section{Introduction}

The idea that the notion of Social Status has relevant consequences on economic activities goes back at least to Adam Smith and, after the work of Veblen (1912), several studies started to inquire the effects of status-seeking behavior on economic policies (see for instance Duesenberry (1949), Frank (1985)). In a seminal work, Cole et al. (1992) show that when the allocation of non market goods is solved by a ranking device such as social status, the social norm describing the way in which status is acquired induces preferences for relative consumption which are absent from individuals underlying deep preferences. ${ }^{1}$ This consideration brings back into the economic analysis, in the spirit of Karl Marx, the significance of the relationship between social organization and economic outcomes.

On the light of these observations, Corneo and Gruner (2000) investigate the relationship between the social organization and redistributive preferences in the presence of status-seeking behavior. In particular, they show that when inequality has an informational value for social decisions, more conservative redistributive policies might be preferred in equilibrium despite the inequality in the distribution of wealth. In their model, individuals have preferences over the other individuals they have to be matched with and the most desirable individuals are those at the top of the distribution of wealth. Therefore, a conservative redistributive policy allows consumption signals to be sufficiently informative about individuals ranking in the society allowing matching to occur between individuals with similar characteristics. Other recent work investigated the consequences of status-seeking behavior on economic and social conditions. Levy and Razin (2015) study the effect of social status considerations on redistributive preferences and they find that, when sorting is costly, rich individuals may prefer more egalitarian redistributive policies. On the same line, Koenig et al. (2017) show that when status is signaled through the consumption of private rather than public goods, the rich may prefer a larger provision of public goods as it fuels the signaling power of the private good they acquire through the market. In a related work, Gallice and Grillo (2018a) consider the case in which status differs across two dimensions, relative standing and social class, and they show that some members of the working class may oppose redistribution, while, at the same time, some members of the elite may favor it. Bilancini and Boncinelli (2018) instead investigate the desirability of income taxes when the objective is to mitigate status consumption generated by people's relative standing concerns. Differently, Gallice and Grillo (2018b) study whether social concerns increase or decrease income and educational inequality when status also depends on the relative individual's achievement in education. ${ }^{2}$

In this article, I extend Corneo and Gruner (2000) work allowing individuals to signal their relative standing through the consumption of a conspicuous good as in Bilancini and Boncinelli (2012). The model develops as follows. There are three social classes, the low class, the medium class and the high class. Individuals who belong to different classes differ in their wealth en-

\footnotetext{
1 Deep preferences are those preferences which can be thought to be hardwired in humans brain thanks to the working of evolutionary forces whereas reduced form preferences depend on the nature of the social interactions individuals are concerned with. See Postlewaite (1998)

2 See Gallice (2018) for a recent survey about social status, redistribution and optimal taxation.
} 
dowment which determines their matching value. ${ }^{3}$ Individuals receive utility from consumption and matching with another individual. Since wealth is private information, I allow individuals to consume a conspicuous good in order to show their relative standing. Thus, matching is arranged according to the way in which individuals strategically display their relative standing. At the beginning of the game, a linear marginal tax rate of redistribution is selected by majority voting and I study to what extent the matching arrangement affects classes redistributive preferences. Since the notion of status employed is ordinal, ${ }^{4}$ economic equality fuels status competition and therefore conspicuous consumption (Hopkins and Kornienko, 2004, 2009). Inasmuch as classes ability to signal their status depends on their relative wealth endowment, these concerns endogenously affect the extent of redistribution. In the spirit of Cole et al. (1992), I observe that there exist different matching arrangements which are supported by specific norms describing the way in which classes separate themselves by means of different conspicuous consumption levels. ${ }^{5}$ In particular, a norm is an equilibrium of the signaling stage and I refer to a social arrangement as an equilibrium of the signaling stage along with its matching outcome. Therefore, I show that keeping constant the characteristics of the economy, the equilibrium marginal tax rate of redistribution that wins any pairwise comparison differs across arrangements. In some arrangements, the majority of voters show no relative concerns for wealth and full redistribution is voted in equilibrium. In some others, the desire to maintain or to increase social success depresses the extent of redistribution. Nevertheless, those different arrangements are all compatible with the underlying characteristics of the economy. As stressed by Cole et al. (1992), it is therefore fundamental to understand the origin of the social norm ruling the society. A wide stream of literature tackles this quest from an evolutionary perspective. On the contrary, in this work I advance the following considerations. The first is that a marginal tax rate of redistribution conveys more information than simply the size of the net transfer classes receive from the redistributive policies. In particular, individuals know which marginal tax rate of redistribution would be voted in each social arrangement. Thus, the second consideration is that the scope of the voting game, which takes place at the beginning of the game, might be larger than simply determining the equilibrium redistributive policy. Therefore, I solve the equilibrium, i.e., the social arrangement, selection problem, by means of aggregating classes preferences over economic outcomes according to majority rule. That is to say, the selected social arrangement is grounded on some form of class representation which safeguards the interests of the majority. In this sense, the medium class, who lies in between the other classes, has two ways through which she can increase her social success. She can either prefer to fully redistribute wealth, with the consequence that status consumption shrinks to zero and matching occurs randomly within the population, or she can prefer to reach a matching agreement with the high class in exchange of the lowest equilibrium marginal tax rate of redistribution. In this arrangement, the fear of the rise

\footnotetext{
3 One can think about the low class as the "poor" and the high class as the "rich". Thus, the matching value reflects the individual position in the distribution of wealth and its class of origin.

4 Bilancini and Boncinelli $(2008,2012,2018)$ stress that the shape of status concerns is as much as relevant as the arrangement itself. For example, when status has cardinal considerations, i.e., it depends not only on the ordinal ranking but also on the distance from the others, then equality may reduce status consumption.

5 In Cole et al. (1992) status is either determined by the position in the distribution of wealth or inherited. In this article, status reflects the position in the distribution of wealth whereas the multiplicity of conspicuous consumption levels supports different matching arrangements.
} 
of the low class brings the society towards the organization in which the ostracism against the low class, i.e., the poor, is maximized.

\section{The Model}

There are two identical unit-mass continua of individuals who are matched into pairs. For simplicity the continua are symmetric in what follows. A generic individual $i \in[0,1]$ belongs to one of three classes: $L$ or the low class, $M$ or the medium class, $H$ or the high class. ${ }^{6}$ An individual in class $K \in \mathscr{K}=\{L, M, H\}$ has resources $R_{K} \in \mathbb{R}_{+}$. I assume that each class has the same measure in both continua and that $R_{L}<R_{M}<R_{A}<R_{H}$ where $R_{A}$ denotes the average level of resource in the population. Individuals derive utility from consumption of their post-tax resource level and matching with another individual, that yields payoff $v(\cdot)$. Individuals share the same preferences described by the utility function $U=u(c)+v(\mu)$ where $c$ is consumption and $\mu \in[0,1]$ denotes a matching partner. ${ }^{7}$ I assume that $u$ satisfies standard conditions, namely $u(\cdot)^{\prime}>0, u^{\prime \prime}(\cdot)<0$. The partner of individual $i$ is individual $\mu(i)=j$ and $v(j)$ is the payoff derived from matching with $j$. The payoff derived from matching with an individual belonging to class $K$ is $k=l, m, h$ with $l<m<h$. Corneo and Gruner (2000) refer to $k$ as the social value of the class which I will refer to as social status. Notice that it is implicitly assumed a positive relationship between social status and wealth, the latter measured by $R_{K}$. Furthermore, matching values are stable in the sense that an exogenous variation of resources does not affect matching values. Although the distribution of resources and matching payoffs are common knowledge, the class of any single individual is a private information. Therefore, individuals signal their matching values by consuming a status good $x$. The cost of showing $x$ however is $f(x)$ with $f(0)=0, f^{\prime}(\cdot)>0$ and $f^{\prime \prime}(\cdot) \leq 0$ which means that the status signal is produced by a concave technology and, thus, the resources spent are convex. $^{8}$

The model has three stages. In the first stage, a marginal tax rate $t \in[0,1]$ is determined by majority voting. ${ }^{9}$ The net tax paid by an individual belonging to class $K$ is $T_{K}=t\left(R_{A}-R_{K}\right)$. In the second stage individuals make their consumption decisions and signal their class. Thus, for a generic individual $i \in K, i$ 's post-tax consumption net of conspicuous consumption, is

$$
c_{i}=R_{K}+t\left(R_{A}-R_{K}\right)-f\left(x_{i}\right) .
$$

Finally, in the third stage individuals are matched into pairs and payoffs are realized.

Intuitively, all individuals would prefer to be matched with a member of the high class who gives the largest matching payoff $v(j \in H)=h$. Nevertheless, matching values are private information and have to be signaled through the consumption of the status good. Since individuals

6 In particular, let $K=L, M, H$ be a subset of $[0,1]$. Then, for all classes, $K \subset[0,1], K \cap K^{\prime}=\emptyset$ for any $K \neq K^{\prime}$ and $L \cup M \cup H=[0,1]$.

7 It is worth to stress that all individuals start with the same preferences whereas relative position concerns are induced by the matching outcome.

8 The assumption is made for technical reasons as it ensures the existence of interior solutions. I discuss the assumption in section 4.

9 For simplicity, I restrict the attention to linear taxes which are equally redistributed to the individuals. 
in different classes posses different resources, individuals in different classes are able to send different signals that could lead to different matching outcomes. Therefore, the choice of the marginal tax rate of redistribution affects the extent of conspicuous consumption by reducing (increasing) the inequality in the post-tax distribution of wealth. Equilibrium is determined by backward induction. In particular, in the third stage, I focus the attention on voluntary matching. Following Cole et al. (1992), a matching rule $\mu:[0,1] \rightarrow[0,1]$ is voluntary if

- no pair prefers to be matched with each other rather than with their matches, that is to say, there does not exist an $i \neq j$ such that $E_{\rho}\left[v(\mu(j)) \mid x_{\mu(j)}\right]>E_{\rho}\left[v(\mu(i)) \mid x_{\mu(i)}\right]$ and $E_{\rho}\left[v(i) \mid x_{i}\right]>$ $E_{\rho}\left[v(j) \mid x_{j}\right]$;

- all individuals receive a partner. ${ }^{10}$

Notice that individuals just observe conspicuous consumption signals from which they form the belief $\rho$ about signal $x$ being sent by an individual belonging to class $K \in \mathscr{K}$. Hence, the expected payoff from matching is taken with respect to the belief function $\rho: \mathbb{R}_{+} \rightarrow[0,1]^{3}$, such that, for all possible signals, $\sum_{K \in\{L, M, H\}} \operatorname{Pr}\left(i \in K \mid x_{i}\right)=1$ and $\operatorname{Pr}\left(i \in K \mid x_{i}\right)=\rho\left(K \mid x_{i}\right)$. Then, $\mu$ pairs individuals according to their signals given the beliefs $\rho$ they may possibly hold.

In the signaling stage, equilibrium is determined by an optimal conspicuous consumption level and a belief function. ${ }^{11}$ Formally, every individual faces the following problem

$$
\max _{x_{i}, f\left(x_{i}\right) \leq R_{i}+T_{i}} u\left(R_{i}+T_{i}-f\left(x_{i}\right)\right)+E_{\rho}\left[v(\mu(i)) \mid x_{i}\right] .
$$

Therefore, in the signaling stage, equilibrium is a pair $\left(\left(x_{K}^{*}\right)_{K \in \mathscr{K}}, \rho\right)$ such that, for all individuals $i \in K$ and for all $x_{i} \neq x_{K}^{*}$

$$
u\left(R_{K}+T_{K}-f\left(x_{K}^{*}\right)\right)+E_{\rho}\left[v\left(\mu(i \in K) \mid x_{K}^{*}\right] \geq u\left(R_{K}+T_{K}-f\left(x_{i}\right)\right)+E_{\rho}\left[v\left(\mu(i \in K) \mid x_{i}\right]\right.\right.
$$

and beliefs are consistent, that is to say, beliefs are derived by Bayes rules whenever possible and unrestricted otherwise. This implies that:

a) if $x_{L}^{*} \neq x_{M}^{*} \neq x_{H}^{*}$ then $\operatorname{Pr}\left(j \in K \mid x_{K}^{*}\right)=1, K=L, M, H$;

b) if $x_{L}^{*}=x_{M}^{*}=x_{H}^{*}$ then $\operatorname{Pr}\left(j \in K \mid x_{K}^{*}\right)=\frac{1}{3}, K=L, M, H$;

c) if $x_{L}^{*}=x_{M}^{*} \neq x_{H}^{*}$ then $\operatorname{Pr}\left(j \in H \mid x_{H}^{*}\right)=1, \operatorname{Pr}\left(j \in K \mid x_{K}^{*}\right)=1 / 2, K=L, M$;

d) if $x_{L}^{*} \neq x_{M}^{*}=x_{H}^{*}$ then $\operatorname{Pr}\left(j \in L \mid x_{L}^{*}\right)=1, \operatorname{Pr}\left(j \in K \mid x_{K}^{*}\right)=1 / 2, K=M, H$.

e) if $x \neq x_{K}^{*}, K=L, M, H$, then $\rho$ is unrestricted.

Notice that, since $\rho$ can assign any belief to out of equilibrium signals, this freedom naturally leads to a multiplicity of both separating and pooling equilibrium. To restrict the set of plausible off of equilibrium beliefs I apply the Intuitive Criterion refinement (see Cho and Kreps (1987) for a formal statement of the refinement). Informally, the Intuitive Criterion states that an off of equilibrium

\footnotetext{
${ }^{10}$ Formally it requires $\mu$ to be measure preserving, see Cole et al. (1992) for details about the matching process.

${ }^{11}$ What is not spent in conspicuous consumption is thus consumed.
} 
message can not be sent by a type of player for which the payoff arising from the off equilibrium message is dominated by its equilibrium payoff. Therefore, individuals will assign probability 0 to this message being sent by a such a type. This restriction on off of equilibrium beliefs restrict the set of equilibria although it does predict a unique equilibrium. Of particular interest is the least-costly separating equilibrium (Riley, 2001) which entails the following conditions:

$$
\begin{aligned}
& u\left(R_{L}+T_{L}-0\right)+E_{\rho}[v(\cdot) \mid 0]=u\left(R_{L}-f\left(x_{M}^{*}\right)+T_{L}\right)+E_{\rho}\left[v(\cdot) \mid x_{M}^{*}\right], \\
& u\left(R_{M}+T_{M}-f\left(x_{M}^{*}\right)\right)+E_{\rho}\left[v(\cdot) \mid x_{M}^{*}\right]=u\left(R_{M}+T_{M}-f\left(x_{H}^{*}\right)\right)+E_{\rho}\left[v(\cdot) \mid x_{H}^{*}\right] .
\end{aligned}
$$

The meaning of conditions (3) and (4) is the following. In equilibrium $x_{M}^{*}$ is the minimum level of conspicuous consumption of the medium class that discourages individuals of the low class to mimic their behavior. Analogously, $x_{H}^{*}$ prevents mimicking behavior from the medium class. Then, the Intuitive Criterion restricts the beliefs individuals may hold following the observation of out of equilibrium signals. For instance, suppose that the out of equilibrium signal $x \in\left(x_{M}^{*}, x_{H}^{*}\right)$ is observed. Then, individuals assign probability 0 to this signal being sent by an individual of the low or the high class. This follows from the fact that, from (3), if an individual of the low class were to make such a deviation she would receive a payoff strictly dominated by her equilibrium payoff. Differently, an individual of the high class cannot gain by such a deviation and, therefore, $\rho$ assigns probability 1 to the out of equilibrium signal coming from an individual of the medium class by mistake. ${ }^{12}$

I take the previous conditions as a reference point to analyze the reduced form preferences over the marginal tax rate in the first stage. ${ }^{13}$ In the following I will also consider the best pooling equilibrium, the pooling in which $x_{K}^{*}=0$ for all $K$ and the least costly semi pooling equilibria. In the first semi pooling equilibrium, the low class pools with the medium class and, therefore, the conspicuous consumption strategy of the high class satisfies

$$
\left.u\left(R_{M}+T_{M}-0\right)+E_{\rho}[v(\cdot) \mid 0]=u\left(R_{M}+T_{M}-f\left(x_{H}^{l, m}\right)\right)+E_{\rho}\left[v(\cdot) \mid x_{H}^{l, m}\right)\right] .
$$

In the second semi pooling, the medium class pools with the high class and the conspicuous consumption strategy then solves

$$
u\left(R_{L}+T_{L}-0\right)+E_{\rho}[v(\cdot) \mid 0]=u\left(R_{L}+T_{L}-f\left(x_{M}^{m, h}\right)\right)+E_{\rho}\left[v(\cdot) \mid x_{M}^{m, h}\right], x_{M}^{m, h}=x_{H}^{m, h} .
$$

Notice that the semi pooling are least costly in the sense that the signaling class (or classes) burns in conspicuous consumption the minimum amount which sustains the equilibrium.

\section{Matching Outcomes}

I start with the first backward induction step discussing the matching outcome that occurs at the end of the game after the voting and the signaling stages. The matching outcome depends on the expected value from matching $E_{\rho}\left[v(j) \mid x_{j}\right]$ individuals assign to any individual $j \in[0,1]$ who

\footnotetext{
12 On the contrary, other beliefs may be compatible with deviations from the conspicuous consumption strategy breaking down the equilibrium.

${ }^{13}$ Throughout the paper I restrict the attention to pure strategies for the ease of exposition.
} 
sent the signal $x_{j}$. There are essentially three cases to consider. In the first case, all individuals send the same signal $x_{P}$. Thus, for all $j \in[0,1]$, for all $K, \rho\left(K \mid x_{P}\right)=1 / 3$ and $E_{\rho}\left[v(j) \mid x_{P}\right]=s$ where $s=\frac{l+m+h}{3}$ is the average matching value in the population. Then, the unique matching outcome is the one in which individuals are randomly matched across the continua. In the second case, $x_{L} \neq x_{M} \neq x_{H}$ and $\rho\left(K \mid x_{K}\right)=1$ for all classes. Since the expected values $E_{\rho}\left[v(j) \mid x_{K}\right]=k^{\prime} \in$ $\{l, m, h\}$ can be ordered according to the matching value associated to each signaling class, then the matching rule $\mu$ is assortative on $E_{\rho}\left[v(j) \mid x_{K}\right]$. That is to say, for all $i \in K, \mu\left(i_{\left(x_{K}\right)}\right)=j_{\left(x_{K}\right)} \in K$. To prove the claim that the matching rule is assortative, consider instead any measure preserving matching rule $\mu^{\prime}$ that prescribes to some $i$ a partner $\mu^{\prime}(i)=j$ who sent a different signal. Since the continua are symmetric, there are at least two pairs of matched individuals $(i, j)$ such that each pair is either $\left(i \in M, \mu^{\prime}(i) \in L\right)$ or $\left(i \in M, \mu^{\prime}(i) \in H\right)$ or $\left(i \in L, \mu^{\prime}(i) \in H\right)$. Inside each pair however, the individuals who sent the signal $x_{K}^{\prime}$ with the largest expected value $k^{\prime}$ would prefer to be matched together rather than with their partner $\mu^{\prime}$. Hence, any $\mu^{\prime}$ that is not assortative on $E_{\rho}\left[v(j) \mid x_{K}\right]$ cannot be stable. In the third case, at least two classes send the same signal $x_{P}$ and the remaining class sends the signal $x_{C}$. Then, $\rho\left(C \mid x_{C}\right)=1, \rho\left(K \mid x_{P}\right)=\rho\left(K^{\prime} \mid x_{P}\right)=1 / 2, K \neq K^{\prime} \neq C$, and the matching rule is restricted on the expected values of the two signals, $E_{\rho}\left[v(j) \mid x_{C}\right] \in\{l, m, h\}$ and $E_{\rho}\left[v(j) \mid x_{P}\right]=\bar{k}$ where $\bar{k}$ is the average matching value between the classes sending signal $x_{P}$. Repeating the previous argument, the stable matching rule $\mu$ is assortative.

Therefore, there are stable matching outcomes in which individuals who belong to different classes are paired together. However, since the matching outcome depends on the conspicuous consumption strategy played in the signaling stage, following Cole et al. (1992), I will refer to a social arrangement as an equilibrium of the signaling stage along with its stable matching outcome. Indeed, the equilibrium signaling strategy describes the way in which matching is arranged among the different classes as pointed out in the following section.

\section{Social Arrangements and Redistributive Preferences}

The purpose of this section is to characterize the equilibrium marginal rate of redistribution in each social arrangement.

To begin with, I consider the best pooling social arrangement. In the best pooling, all individuals send the same equilibrium signal $x_{K}^{*}=0$ and the matching outcome is random, i.e., $E_{\rho}\left[v \mid x_{K}^{*}\right]=s$. It is hence linear to conclude that in the best pooling social arrangement there is a unique equilibrium marginal tax rate $t^{P}=1$ which is always preferred by the lower and the medium class who form the winning majority.

Proposition 1. In the best pooling social arrangement, the unique equilibrium marginal rate of redistribution is $t^{P}=1$ and all individuals have payoff

$$
U^{P}=u\left(R_{A}\right)+s
$$

Proof. When $x_{K}^{*}=0$ for all classes, the equilibrium payoff of the high class strictly decreases in the marginal tax rate of redistribution. On the contrary, the equilibrium payoff of the low and the medium class strictly increases in $t$. Therefore, the marginal tax rate $t=1$ wins any pairwise 


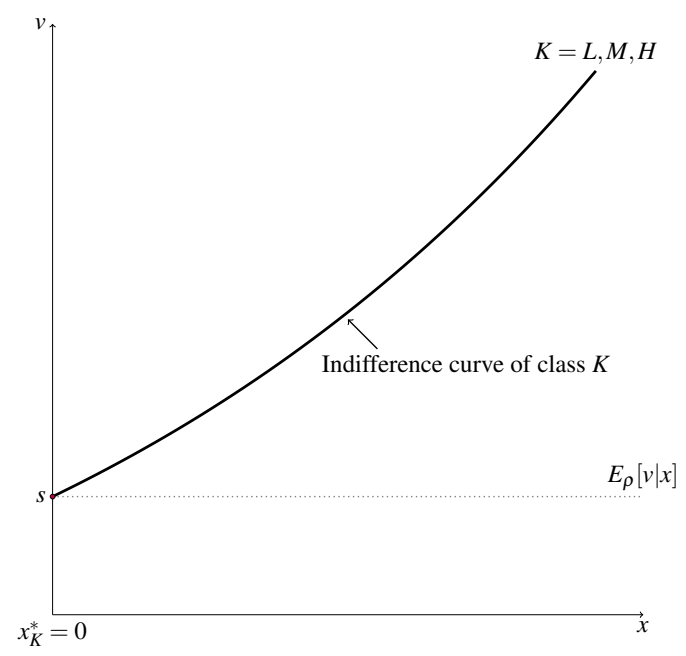

Figure 1: Best pooling social arrangement. All individuals and all classes lie on the same indifference curve. The dotted line is the expected value from matching given beliefs $\rho$. It is constant and equal to $s$ for any deviation will always be matched with an individual with $x_{K}^{*}=0$.

comparison and it is therefore the unique Condorcet winner of the voting stage. Thus, at $t^{P}$ all individuals post-tax resource level is set to $R_{A}$ and, since the matching outcome is random, for any individual the equilibrium payoff in the social arrangement is the utility from consumption of the post tax resource level $u\left(R_{A}\right)$ plus the expected value from matching, that is $U^{P}=u\left(R_{A}\right)+s$. Finally, notice that no deviations are profitable. Consider any deviation $x>0$. Regardless from the beliefs $\rho$ individuals may hold, the match could only happen with another individual with conspicuous consumption level $x^{*}=0 .{ }^{14}$

The previous Proposition stresses the fact that the best pooling social arrangement does not induce concerns for relative position in individuals reduced form preferences. Inasmuch as all individuals adopt the same conspicuous consumption strategy, the matching outcome is random. Thus, since $R_{A}>R_{M}>R_{L}$, the majority of voters (the low and the medium class) strictly prefers to fully redistribute wealth and, in equilibrium, all individuals lie on the same indifference curve with payoff $U^{P}$ as shown in Figure 1.

Nonetheless, the differences in the conspicuous consumption strategy that arise across different social arrangements play a major role in shaping the redistributive extent of a social arrangement. I deal now with the natural case in which classes fully separate in the best separating social arrangement. Whenever individuals engage in conspicuous consumption in order to signal their matching values, in the best separating equilibrium individuals in the low class invest all their resources in consumption. On the contrary, the medium and the high class invest part of their resources in conspicuous consumption in such a way that the other classes are discouraged to mimic their own status consumption. That is to say, classes separate by means of the least costly status consumption strategy implicitly defined in (3) and (4). From the fact that $R_{L}<R_{M}<$ $R_{H}, 0=x_{L}^{*}<x_{M}^{*}<x_{H}^{*}$, matching is assortative and every individual $i \in K$ is matched with an

\footnotetext{
14 This follows from the fact that the matching function $\mu$ sorts individuals according to their signals inasmuch as each individual's payoff is private information.
} 


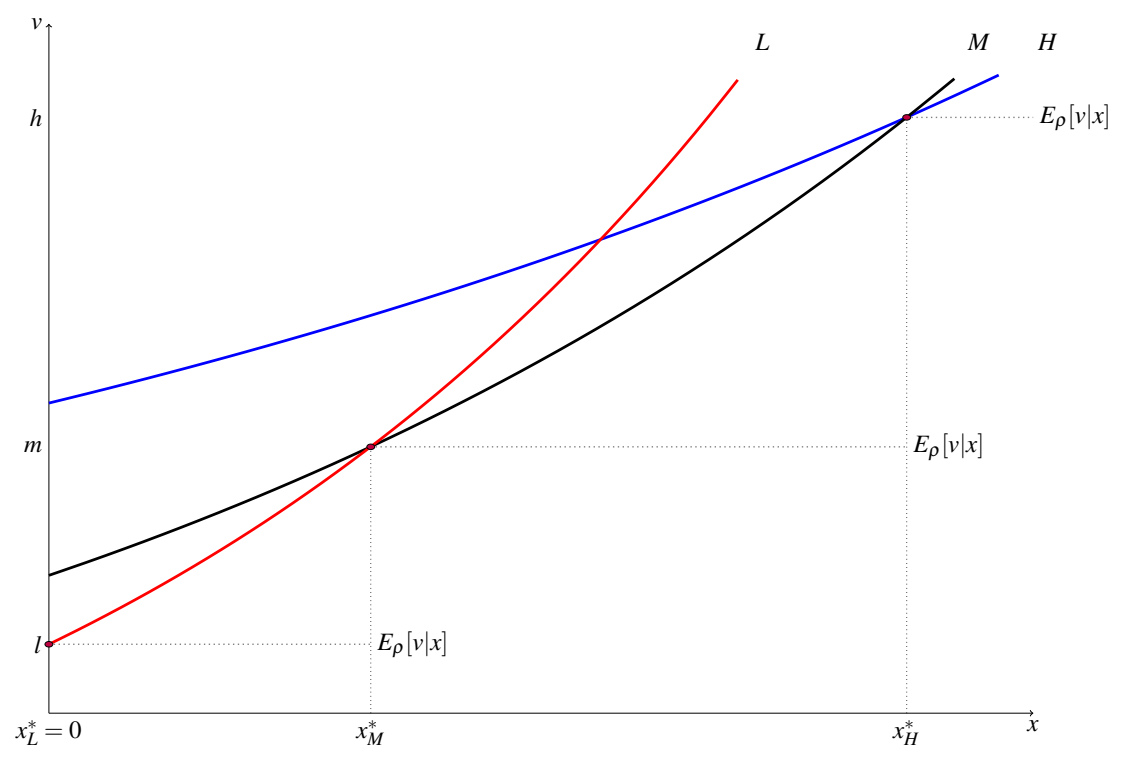

Figure 2: Best separating social arrangement. Since the matching rule is assortative, the expected value from matching is a step line function. Furthermore, since $R_{L}<R_{M}<R_{H}$ the low class lies on the steeper indifference curve.

individual who belongs to the same social class. In particular, $E_{\rho}\left[v \mid x_{K}\right]=k$. The social arrangement equilibrium is depicted in Figure 2. Nevertheless, the choice of the marginal tax rate influences the size of each transfer $T_{K}$ and thus classes' ability to signal their status. Not surprisingly, the conspicuous consumption strategy $x_{C}^{*}(\cdot), C=M, H$, is strictly increasing in the marginal tax rate of redistribution $t$.

Lemma 1. In the best separating social arrangement, the conspicuous consumption strategy of the medium and the high class is strictly increasing in the marginal tax rate of redistribution $t$.

Proof. Totally differentiating (3) with respect to $t$ one obtains

$$
\frac{d}{d t} x_{M}^{*}=\frac{\left(R_{A}-R_{L}\right)}{f^{\prime}\left(x_{M}^{*}\right)}\left[1-\frac{u^{\prime}\left(R_{L}+T_{L}\right)}{u^{\prime}\left(R_{L}-f\left(x_{M}^{*}\right)+T_{L}\right)}\right]>0
$$

which is positive due to the concavity of $u(\cdot)$, i.e., $u^{\prime}\left(R_{L}-f\left(x_{M}^{*}\right)+T_{L}\right)>u^{\prime}\left(R_{L}+T_{L}\right)$. Analogously, from (4),

$$
\begin{aligned}
\frac{d}{d t} x_{H}^{*}=\frac{\left(R_{A}-R_{M}\right)}{f^{\prime}\left(x_{H}^{*}\right)}\left[1-\frac{u^{\prime}\left(R_{M}+T_{M}-f\left(x_{M}^{*}\right)\right)}{u^{\prime}\left(R_{M}+T_{M}-f\left(x_{H}^{*}\right)\right)}\right] & \\
& +\frac{u^{\prime}\left(R_{M}+T_{M}-f\left(x_{M}^{*}\right)\right)}{u^{\prime}\left(R_{M}+T_{M}-f\left(x_{H}^{*}\right)\right)} \frac{d}{d t} f\left(x_{M}^{*}\right)>0 .
\end{aligned}
$$

Thus, for $K=M, H, \frac{d}{d t} x_{K}^{*}>0$.

In order to determine the equilibrium marginal tax rate of redistribution I now discuss classes redistributive preferences. Although the status consumption strategy is increasing in the marginal tax rate of redistribution for both the medium and the high class, the high class' transfer is always 
negative and hence the high class equilibrium payoff is strictly decreasing in $t$. Therefore, $t=0$ is the preferred marginal rate of redistribution of the high class. On the contrary, the low class always prefer to vote for largest marginal tax rate of redistribution whereas the medium class preferred rate solves a trade off between a larger transfer $T_{M}$ and an increased status consumption. The medium class preferred marginal tax rate of redistribution $\hat{t}$ is defined as

$$
\hat{t}=\operatorname{argmax}_{t \in[0,1]} u\left(R_{M}+T_{M}-f\left(x_{M}^{*}(t)\right)\right)+m
$$

and, if $\hat{t}$ is interior, it satisfies the first order condition

$$
R_{A}-R_{M}=\frac{d}{d t} f\left(x_{M}^{*}(\hat{t})\right)
$$

which says that the marginal benefit of redistribution has to be equal to the marginal cost, i.e., the increased status consumption. I now assume that $f$ is sufficiently concave such that $x_{M}^{*}(\cdot)$ is convex so that an interior solution exists as pointed out in the following example.

Example 1. Let $u(c)=\log (c)$ and $f(x)=x$. Then, solving (3) for $x_{M}^{*}$ one obtains

$$
x_{M}^{*}=c_{L}(t)\left(1-\frac{1}{e^{m-l}}\right)
$$

Since $x_{M}^{*}(\cdot)$ is linear in $t$, the preferred marginal rate lies on the extremes. Consider however the case in which $f(x)=\sqrt{x}$. Then:

$$
x_{M}^{*}=\left(c_{L}(t)\right)^{2}\left(1-\frac{1}{e^{m-l}}\right)^{2}
$$

which is convex and thus $\hat{t} \in[0,1]$.

The concavity of $f$ implies that residual consumption decreases less and less as $x$ increases (see Figure 3). The assumption is plausible in different situations. For example, it reflects the fact that wealthier individuals may have an advantage in the exhibition of their relative standing. It also applies to the cases in which the consumption of status goods have an intrinsic value. A private

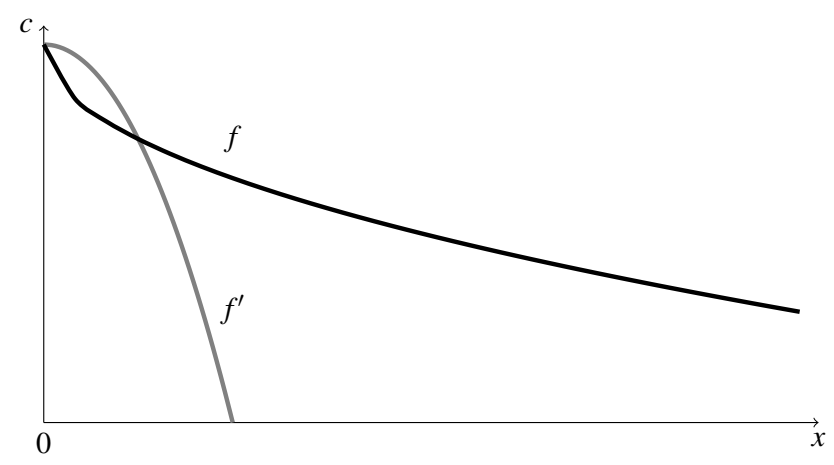

Figure 3: Residual consumption as a function of $x$ for different functions $f$. In the picture, $f$ is concave whereas $f^{\prime}$ is convex and $c$ decreases faster as $x$ increases. 
schooling choice may signal status and have a larger intrinsic value with respect the choice of enrolling in a public school. ${ }^{15}$ Nevertheless, in some other cases it is reasonable to expect residual consumption decreasing more and more as conspicuous consumption increases. For instance, an increase in status consumption may push an individual to disregard other types of consumption in the attempt to maintain her relative standing. ${ }^{16}$ In those cases, it is not reasonable to assume $f$ being concave and the preferred marginal tax rate of redistribution would lie on the corner.

In Corneo and Gruner (2000) the medium class preferred marginal tax rate of redistribution solves a trade-off between more consumption and less social success. On the contrary, when signals are costly, the transfer received by the medium class has to optimally balance the cost of separation which prevents matching to occur between individuals of different classes. Therefore, given the preferences of the other classes, the equilibrium marginal tax rate of redistribution in the best separating social arrangement is entirely determined by the medium class' preferences.

Proposition 2. In the best separating social arrangement, the equilibrium marginal tax rate of redistribution is the medium class preferred marginal rate $\hat{t}=t^{*} \in[0,1]$. Moreover, if $\hat{t}=1$, in equilibrium all individuals receive the same payoff

$$
U^{S}=u\left(R_{A}\right)+l=u\left(R_{A}-f\left(x_{M}^{*}\right)\right)+m=u\left(R_{A}-f\left(x_{H}^{*}\right)\right)+h<U^{P} .
$$

Proof. Inasmuch as the low class equilibrium payoff is strictly increasing in $t$ and the high class equilibrium payoff is strictly decreasing in $t$, in equilibrium the rate $\hat{t}$ wins any pairwise comparison and it is therefore the unique Condorcet winner. The medium class preferred rate solves the problem

$$
\hat{t}=\operatorname{argmax}_{t \in[0,1]} u\left(R_{M}+T_{M}-f\left(x_{M}^{*}\right)\right)+m
$$

where

$$
x_{M}^{*}=f^{-1}\left(R_{L}+T_{L}-u^{-1}\left(u\left(R_{L}+T_{L}\right)+l-m\right)\right) .
$$

Hence, given the convexity of $x_{M}^{*}(\cdot)$, for some economies there exists an interior $\hat{t} \in(0,1)$ such that $R_{A}-R_{M}=\frac{d}{d t} f\left(x_{M}^{*}(\hat{t})\right)$. Finally, if $\hat{t}=1$, then all individuals post-tax resource level is set to $R_{A}$. The best separating condition implies that all individuals receive the same equilibrium payoff, the fact that $U^{P}>U^{S}$ follows from $l<s$.

Proposition 2 stresses the fact that the equilibrium marginal tax rate of redistribution is determined by the medium class' preferences and that, in the best separating equilibrium, the medium class may prefer any rate $t \in[0,1]$. In any interior equilibrium, the preferred marginal tax rate of redistribution of the medium class depends on the separating condition (3) and the first order condition (9). Thus, differently from Corneo and Gruner (2000) in which the extent of redistribution is positively correlated with the value differential $h-m$ and negatively correlated with the value differential $m-l$, in the best separating equilibrium the medium class shows only a downward looking concern over the low class which is kept away from the matching outcome by means of the status consumption strategy $x_{M}^{*}(\cdot)$. To study the effect of inequality on the equilibrium outcome, I define economic inequality as the differential $R_{H}-R_{L}$ and social inequality as the

\footnotetext{
${ }^{15}$ See Koenig et al. (2017); Gallice and Grillo (2018a) for related arguments.

${ }^{16} \mathrm{I}$ thank an anonymous referee for having advanced this point and suggested the example I reported.
} 
differential $h-l$. I consider therefore a change of the differentials which nevertheless keep the averages and the medium class condition constant. ${ }^{17}$

Lemma 2. In an interior equilibrium of the best separating social arrangement, the equilibrium marginal tax rate of redistribution always decreases with social inequality. The equilibrium marginal tax rate of redistribution decreases with economic inequality only if the differential $R_{A}-R_{M}$ is sufficiently large.

Proof. In an interior equilibrium the medium class status consumption strategy $x_{M}^{*}(\cdot)$ depends on the resource level and the matching value of the low class. On the contrary, the matching value of the high class has no effect on $x_{M}^{*}(\cdot)$ whereas $R_{H}$ has an indirect effect through $R_{A}$. Thus, from the point of view of the medium class, any increase in the differentials $R_{H}-R_{L}$ or $h-l$ which leaves the respective averages unchanged, is equivalent to a decrease of either $R_{L}$ or $l$. From the separating condition

$$
u\left(R_{L}+T_{L}\right)+l=u\left(R_{L}+T_{L}-f\left(x_{M}^{*}\right)\right)+m
$$

it follows that the status consumption strategy of the medium class depends negatively on $l$ and positively on $R_{L}$. A positive shock to the status of the low class reduces the medium class status consumption. Indeed, in order to preserve the equality, an increase of $l$ has to be compensated, on the right side, by a decrease of $x_{M}^{*}$. Similarly, an increase of $R_{L}$ fuels status consumption. Implicitly differentiating $x_{M}^{*}$ with respect to $R_{L}$ one obtains

$$
\frac{d}{d R_{L}} x_{M}^{*}=\frac{(1-t)}{f^{\prime}\left(x_{M}^{*}\right)} \frac{u^{\prime}\left(c_{L}-f\left(x_{M}^{*}\right)\right)-u^{\prime}\left(c_{L}\right)}{u^{\prime}\left(c_{L}-f\left(x_{M}^{*}\right)\right)}>0
$$

whose sign follows from the concavity of $u(\cdot)$. The effect of social or economic inequality on the preferred marginal tax rate of redistribution of the medium class is then pinned down by the marginal cost condition (9). When $l$ increases, the marginal benefit from redistribution remains constant whereas status consumption decreases. From the convexity of $x_{M}^{*}(\cdot)$, the marginal cost of redistribution decreases with $l$ and, as a consequence, $\hat{t}$ increases in $l$. Therefore, $\hat{t}$ decreases with social inequality. Consider now an increase of economic inequality so that $R_{L}$ decreases to $R_{L}^{\prime}$. This has two effects. The first is that status consumption decreases. The second is that, following the decrease of $R_{L}$, the marginal benefit from redistribution of the low class has increased. Since $x_{M}^{*}(\cdot)$ is convex, one must have that

$$
\frac{\partial}{\partial t} x_{M}^{*}\left(R_{L}, t=0\right)>\frac{\partial}{\partial t} x_{M}^{*}\left(R_{L}^{\prime}, t=0\right)
$$

that is to say, the marginal cost when $t=0$ is larger at $R_{L}$ since $R_{L}>R_{L}^{\prime}$. Nevertheless, for the same reason, the marginal cost does not increase in $t$ at the same speed for both values $R_{L}$ and $R_{L}^{\prime}$. In particular, the marginal benefit from redistribution of the lower class decreases in $R_{L}$. Since $t\left(R_{A}-R_{L}\right)<t\left(R_{A}-R_{L}^{\prime}\right)$ the convexity of $x_{M}^{*}(\cdot)$ implies that there exists a threshold value $\tilde{t}$ after

\footnotetext{
${ }^{17}$ As underlined by Corneo and Gruner (2000), economic inequality impacts the equilibrium tax rate in two distinct ways. The first is the classical effect, that is, when inequality increases, the marginal effect of redistribution on the median voter increases. The second effect on the contrary is endogenously derived within the model. In order to separate the effects, it is worth to measure economic inequality with the differential $R_{H}-R_{L}$.
} 


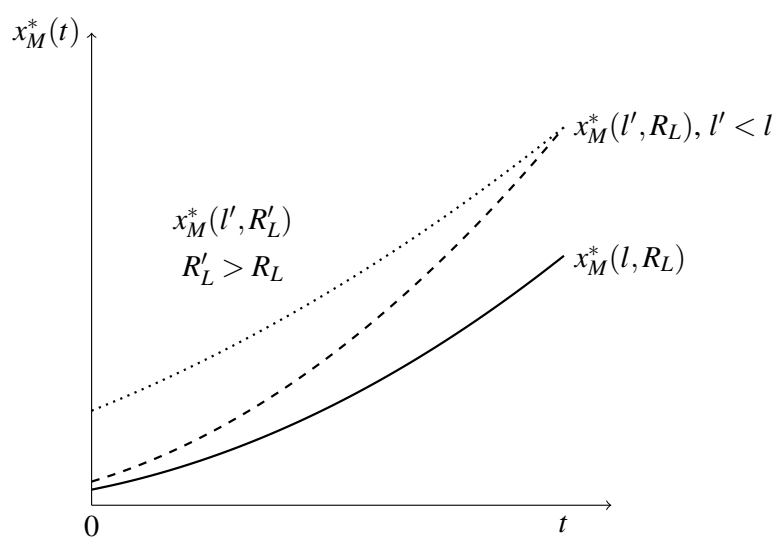

Figure 4: Medium class' conspicuous strategy when $u(c)=\log (c)$ and $f(x)=\sqrt{x}$. Keeping the other parameters constant, for any $t$, an increase of social inequality fuels status consumption. That is, $x_{M}^{*}$ shifts upward following the increase in the differential $h-l$. Analogously, the dotted line shows the upward shift effect on $x_{M}^{*}$ due to an additional increase of $R_{L}$.

which $\frac{\partial}{\partial t} x_{M}^{*}\left(R_{L}^{\prime}, \tilde{t}\right)>\frac{\partial}{\partial t} x_{M}^{*}\left(R_{L}, \tilde{t}\right)$, in other terms $\frac{\partial^{2}}{\partial R_{L} \partial t} x_{M}^{*}\left(t, R_{L}^{\prime}\right)>\frac{\partial^{2}}{\partial R_{L} \partial t} x_{M}^{*}\left(t, R_{L}\right)$. That is to say, the two marginal cost curves eventually cross at $\tilde{t}$. Finally, recall that the change in $R_{L}$ to $R_{L}^{\prime}$ has left the differential $R_{A}-R_{M}$ unchanged. Therefore, $t_{R_{L}^{\prime}}^{*}>t_{R_{L}}^{*}$ only if $\tilde{t}>t_{R_{L}}^{*}$. Since $t_{R_{L}}$ solves the first order condition $R_{A}-R_{M}=\frac{d}{d t} f\left(x_{M}\left(t_{R_{L}}\right)\right)$, the equilibrium marginal tax rate of redistribution decreases with economic inequality only if $R_{A}-R_{M}$ is sufficiently large.

Corneo and Gruner (2000) show that when status is inferred by the imperfect observation of consumption signals, then the equilibrium marginal tax rate of redistribution decreases with economic inequality measured by the differential $R_{H}-R_{L}$. On the contrary, when there is a social norm prescribing the status expenditure of each class in order to preserve separating matching arrangements, then the position of the medium class with respect to the average resource level in the economy determines whether the equilibrium marginal tax rate of redistribution increases or not with economic inequality. ${ }^{18}$ In particular, from the point of view of the medium class, an increase of economic inequality comes with two effects. On the one hand, the decreased signaling ability of the low class reduces the medium class status consumption. On the other hand however, the low class receives a larger transfer at each $t$, i.e., the marginal benefit from redistribution of the low class increases. Which of the effect is stronger determines the change in the equilibrium tax rate. However, if $R_{A}-R_{M}$ is sufficiently large, the second effect is likely to dominate and the equilibrium marginal tax rate decreases with economic inequality. In other terms, when the classic effect which comes along with the redistribution of wealth is strong on the median voter, then it is more likely that an increase of inequality fuels status consumption decreasing the marginal redistribution rate in the economy. Figure 4 and 5 summarize the comparative statics discussed so far. Figure 4 describes how the status consumption strategy of the medium class responds to changes in the conditions of the low class. Figure 5 instead describes the change in the preferred marginal rate of the medium class following analogous shifts in the parameters.

\footnotetext{
${ }^{18}$ Windsteiger (2017) provides a similar result when status concerns induce individuals to misrepresent the income distribution.
} 


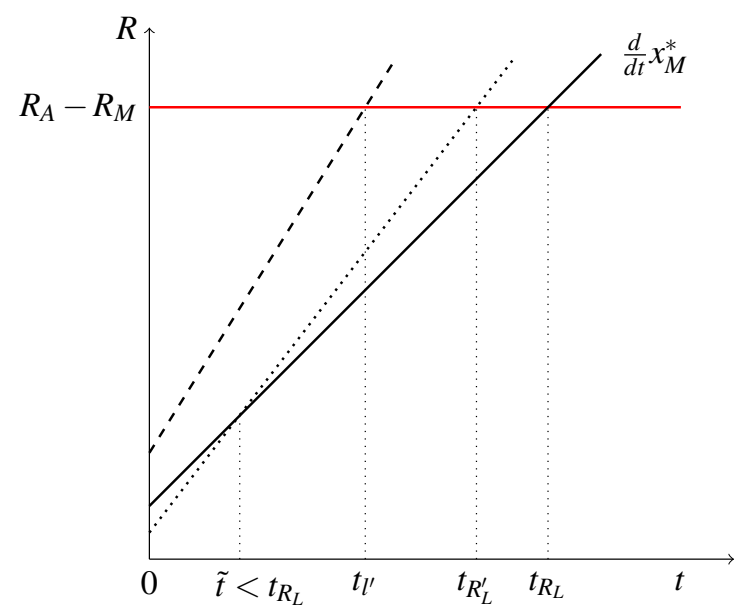

Figure 5: First order condition when $f(x)=\sqrt{x}$. When the change in the parameters leaves $R_{A}-R_{M}$ unaltered, the shift in the preferred marginal rate of the medium class follows the change in the marginal cost of status consumption. The dotted line shows the effect of an increase of $R_{H}-R_{L}$ whereas the dashed line shows the effect of an increase of $h-l$. In this example, $\tilde{t}<t_{R_{L}}$ and therefore the equilibrium marginal tax rate of redistribution decreases with economic inequality.

There are two other social arrangements of interest to consider. In the LM social arrangement the medium class endogenously pools with the poor whereas in the $M H$ social arrangement the rich endogenously pool with the medium class. When two classes pool together, in equilibrium there are just two different signals from which individuals can infer the status of the others. Since at least one signal is sent by two distinct classes, in this case a new endogenous class emerges whose matching value is the average between the two. When the low class pools with the medium class, the expected payoff derived from matching for any individual in the two classes is $\bar{m}=\frac{l+m}{2}<m<s$. On the contrary, when the medium class pools with the high class the average matching payoff of the new class is $\bar{h}=\frac{m+h}{2}>s$ with $m<\bar{h}<h$.

In the $L M$ social arrangement, the high class spend the minimum amount of resources $f\left(x_{H}^{l, m}\right)$ which makes any $i \in L \cup M$ discouraged to mimic the same status consumption. Therefore, since in the least costly pooling $x_{L}^{l, m}=x_{M}^{l, m}=0$, there is a unique equilibrium in which $t^{*}=1$.

Proposition 3. In the LM social arrangement, the unique equilibrium marginal rate of redistribution is $t^{*}=1$ and all individuals have payoff

$$
U^{l, m}=u\left(R_{A}\right)+\bar{m}<U^{P} .
$$

Proof. The least costly semi separating condition implies that

$$
x_{H}^{l, m}>x_{M}^{l, m}=x_{L}^{l, m}=0 .
$$

The assortative matching rule $\mu\left(i \mid x_{i}\right)=j$ with $x_{j}=x_{i}$ is stable since $E_{\rho}\left[v(i) \mid x_{H}^{l, m}\right]=h>\bar{m}=$ $E_{\rho}[v(j) \mid 0] .{ }^{19}$ Insofar $x_{M}^{l, m}=0$, the medium class preferred marginal tax rate is $\hat{t}=1$ and therefore

\footnotetext{
${ }^{19}$ This also implies that there are no profitable deviations in the signaling stage inasmuch as any deviation $x \in\left(0, x_{H}^{l, m}\right)$ would be paired with the signal $x^{*}=0$. Suppose that an individual (she) of the medium class sends the off equilibrium
} 
$\hat{t}$ wins any pairwise comparison in the voting stage being the unique Condorcet winner marginal tax rate. Finally, at $t^{*}=1$ all individuals post tax resource level is set to $R_{A}$ and, from the least costly semi pooling condition, it follows that $U^{l, m}<U^{P}$ since $\bar{m}<s$.

When economic inequality is large, the medium class might prefer the social arrangement in which its individuals experience less social success in exchange of larger transfers from redistribution. To see that the medium class might prefer this social arrangement with respect to the full separating outcome it is sufficient to compare the equilibrium payoffs

$$
U_{M}^{*}=u\left(R_{M}+T_{M}(\hat{t})-f\left(x_{M}^{*}(\hat{t})\right)\right)+m
$$

and

$$
U^{l, m}=u\left(R_{A}\right)+\bar{m} .
$$

When $R_{M}$ is close to $R_{A}$, the marginal benefit of the medium class from redistribution of wealth is lower at any marginal tax rate $t$ and a subtle difference in the matching values $m$ and $l$ might lead the medium class to prefer the separating outcome. On the contrary, when $R_{A}-R_{M}$ is large, the increase in consumption up to $R_{A}$ might compensate the loss in social success which occurs in this social arrangement.

In the latter social arrangement, the extent of redistribution is maximized as in the best pooling equilibrium. Precisely for this reason, in the $L M$ social arrangement the medium class is only concerned about the matching value of the low class.

Lemma 3. In the LM social arrangement an increase of inequality has no effect on the equilibrium marginal rate of redistribution.

The previous lemma follows from the fact that in the specified social arrangement, the medium class equilibrium payoff is $U^{l, m}=u\left(R_{A}\right)+\frac{l+m}{2}$ which is independent from the condition of the higher class. In particular, for all levels of inequality, in the $L M$-social arrangement full redistribution of wealth always occurs which prevents concerns for relative position to arise. Nevertheless, Proposition 3 also stresses that the equilibrium payoff arising in the LM social arrangement is strictly dominated by the payoff arising in the best pooling arrangement inasmuch as, in the best pooling, matching occurs randomly between all individuals and the expected matching payoff is indeed $s>\frac{l+m}{2}$.

In the social arrangement left to discuss in which the medium class mixes with the high class, the medium class invests some of its resources in conspicuous consumption which directly affects its preferences over the marginal tax rate of redistribution. However, it is linear to observe that also in this social arrangement, the political preferences of the medium class are determinant in selecting the equilibrium marginal tax rate. The following Proposition underlines the characteristics of this social arrangement.

signal $x^{\prime} \in\left(0, x_{H}^{l}\right)$ and suppose that $\rho\left(M \mid x^{\prime}\right)=1$. Since the beliefs are such that the type is revealed, no member of the high class would like to match with her rather than their stable matchings. Therefore, she can only be paired with an individual with $x^{*}=0$. In addition, since for any signal $x^{\prime} \geq x_{H}^{l, m}, \rho\left(M \mid x^{\prime}\right)=0$ from the Intuitive Criterion, then the medium class has no profitable deviations. 
Proposition 4. In the MH social arrangement there exists an economy in which the marginal tax rate of redistribution preferred by the medium class $t^{m, h} \in[0,1]$. Then, in an interior equilibrium, for all $t \in[0,1]$ :

$$
x_{M}^{m, h}(t)>x_{M}^{*}(t)
$$

and, in equilibrium, $t^{*}=t^{m, h}=\operatorname{argmax}_{t} U_{M}^{m, h}(t) \leq \hat{t}$.

Proof. The least costly semi separating conditions imply that the medium and the high class pool together by sending the same signal:

$$
x_{M}^{m, h}=x_{H}^{m, h}>x_{L}^{m, h}=0 .
$$

The assortative matching rule $\mu\left(i \mid x_{i}\right)=j$ with $x_{j}=x_{i}$ is stable since $E_{\rho}\left[v(i) \mid x_{H}^{m, h}\right]=\bar{h}>l=$ $E_{\rho}\left[v(j) \mid x_{L}^{m, h}\right] .{ }^{20}$ To see that for all $t, x_{M}^{m, h}(t)>x_{M}^{*}(t)$, it is sufficient to compare the separating conditions:

$$
u\left(R_{L}+T_{L}\right)+l=u\left(R_{L}+T_{L}-f\left(x_{M}^{m, h}\right)\right)+\bar{h}
$$

and

$$
u\left(R_{L}+T_{L}\right)+l=u\left(R_{L}+T_{L}-f\left(x_{M}^{*}\right)\right)+m .
$$

The fact that, for any $t, x_{M}^{m, h}>x_{M}^{*}$ follows from $\bar{h}>m$. It is straightforward to realize that the strategy $x_{M}^{m, h}$ differs from $x_{M}^{*}$ just in the matching payoff of the medium class which is now $\bar{h}$. Therefore, the same considerations of Proposition 2 can be applied to see that there exists an economy in which $t^{m, h} \in(0,1)$. Furthermore, suppose then that for the same economy $t^{*} \in(0,1)$. To see that $t^{m, h} \leq t^{*}$, notice from (7) that $u^{\prime}\left(R_{L}-f\left(x_{M}^{*}\right)+T_{L}\right)<u^{\prime}\left(R_{L}+T_{L}-f\left(x_{M}^{m, h}\right)\right)$. That is, at any $t$, the marginal cost of redistribution is larger in the social arrangement in which the medium class pools with the high class than in the best separating arrangement. Therefore, the preferred rate of the medium class in this arrangement is $t^{m, h} \leq \hat{t}$ and the equality holds whenever $\hat{t}=0$.

In the latter social arrangement, the medium class increases its conspicuous consumption inasmuch the new emerging class with matching value $\bar{h}$ is more distant, with respect the matching value $m$, to the low class. In this social arrangement, from the point of view of the low class, social inequality increases inasmuch the matching value of the relatively new emerging class is larger than the matching payoff the medium class. Furthermore, keeping fixed the parameters of the economy and matching values, in the $M H$ social arrangement, the equilibrium marginal rate of redistribution is the lowest rate voted in equilibrium. This follows from the fact that differently from the best separating arrangement, the matching value of the signaling class is the average between $m$ and $h$ which raises the marginal cost of redistribution since $\bar{h}-l>m-l$.

Lemma 4. In an interior equilibrium of the $M H$ social arrangement, the equilibrium marginal tax rate of redistribution always decreases with social inequality. The equilibrium marginal tax rate of redistribution decreases with economic inequality only if the differential $R_{A}-R_{M}$ is sufficiently large.

${ }^{20}$ Analogously to the previous pooling, deviations from the signaling rule are not profitable. 
Proof. The effect of economic and social inequality follows exactly Lemma 2 with the difference that the status consumption strategy of the medium class depends on $h$ as long as $E\left[v \mid x_{P}^{m, h}\right]=$ $\frac{m+h}{2}=\bar{h}$. Thus, an increase of social inequality increases the matching value of the medium class and decreases that of the low class raising the marginal cost of redistribution.

The multiplicity of social arrangements stems from the multiplicity of equilibria in the signaling stage. Each social arrangement is characterized by a status signaling norm which specifies the status consumption of each class. For this reason, given the description of the economy and its social classes, different marginal tax rate of redistribution would be voted in equilibrium. In the following section I propose an equilibrium selection procedure grounded on majority voting.

\section{Equilibrium Selection}

Given the description of the economy and its classes, it is possible to characterize the equilibrium payoff and the equilibrium marginal tax rate of redistribution in each social arrangement. However, the voting game which takes place at the beginning of the game might have indeed a larger scope than simply deciding the marginal tax rate of redistribution in the economy. Indeed, there are some marginal tax rates which convey the information that they would be the political winners in different social arrangements. When this information is internalized, individuals do not just express their preferences over a marginal tax rate of redistribution but also over a social arrangement. Therefore, in this section I assume that the act of voting does not only concern a marginal tax rate of redistribution but also a social arrangement. Then, majority voting selects not only the equilibrium marginal tax rate of redistribution but also the equilibrium arrangement which is now grounded on a representative dimension of classes' interests.

To begin with, I describe the extended game. At the beginning of the game, for the ease of exposition, the set of marginal tax rate which could be voted is restricted to $\tau=\left\{t^{P}, t^{l, m}, t^{*}, t^{m, h}\right\}$. Thus, $\tau$ is the set that contains the equilibrium marginal tax rate of redistribution that would be voted in each arrangement. ${ }^{21}$ In the extended game, each vote is over a pair made by an equilibrium marginal tax rate of redistribution and the associated social arrangement. At each $t \in \tau$, each class has hence payoff $U_{K}(t)$, which is the equilibrium payoff that any $i \in K$ would get in the arrangement specified by $t \in \tau$. Therefore, the equilibrium of the extended game is the marginal tax rate of redistribution, with its underlying social arrangement, that wins any pairwise comparison. In other words, equilibrium is selected by majority voting. Surprisingly, the medium class is not always politically determinant in selecting the social arrangement and the equilibrium arrangement thus depends on the economic conditions.

Proposition 5. Given $R_{H}>R_{A}>R_{M}>R_{L}$ and $l<m<h$, let $\tau$ be the set of equilibrium marginal tax rate of redistribution which would be independently voted in each social arrangement. Then, in the extended game in which the equilibrium social arrangement is grounded on the preferences of the majority:

\footnotetext{
${ }^{21}$ Although the Intuitive Criterion does not rule out the possibility of separating equilibria that are not least costly, those equilibria are less efficient and would never be voted in equilibrium in the extended game.
} 


\section{i) The LM arrangement is never selected;}

ii) The best poling arrangement is selected if and only if the best pooling is the medium class preferred arrangement;

iii) There exists an economy in which the low class and the high class form the winning majority and the best separating arrangement is selected;

iv) Suppose that $U_{M}^{m, h}\left(t^{m, h}\right)>U_{M}^{P}\left(t^{P}\right)>U_{M}^{*}\left(t^{*}\right)$. Then, if $U_{H}^{*}\left(t^{*}\right)>U_{H}^{m, h}\left(t^{m, h}\right)>U_{H}^{P}\left(t^{P}\right)$, then collective preferences are cyclic. Otherwise, the $M H$ arrangement is selected;

v) If $m \leq s$, then the medium class strictly prefers the best pooling to the best separating arrangement. Nevertheless, the MH social arrangement might be selected even if $m<s$.

Proof. To start with, notice that the low class payoff will always satisfy

$$
U_{L}^{\cdot}\left(t^{P}\right)>U_{L}\left(t^{l, m}\right)>U_{L}^{\cdot}\left(t^{*}\right)>U_{L}^{*}\left(t^{m, h}\right)
$$

Indeed, the payoff of the low class is maximized when $t=1$ and the best pooling is played. The latter inequality follows from the fact that $t^{m, h}<t^{*}$. On the contrary, the medium class and the high class preferences change across economies. Nevertheless, it is possible to rule out the marginal tax rate $t^{l, m}$ being at the top of their preferences. At $t=1$ indeed, all classes prefer the best pooling equilibrium. Therefore, $t^{l, m}$ can never win any pairwise comparison. Furthermore, whereas the medium class may place at the top of its ranking any $t \in \tau$, the high class puts $t^{P}$ always at the bottom of its ranking. To prove ii), it is sufficient to note that, since the high class always rank the best pooling at the bottom, then the pooling arrangement arises if and only if the medium class strictly prefers the best pooling to any other arrangement. To prove iii) and $i v$ ) consider Table 1. Classes preferences are presented in a descending order. In particular, in the left table, Table 1a, classes preferences are such that the unique Condorcet Winner arrangement is the best separating point iii). In the right table instead, Table $1 \mathrm{~b}$, classes preferences exhibit the Condorcet's paradox, i.e., majority preferences are cyclic. Nevertheless, the cycle is solved if either the medium class places $S$ or $P$ at the top of its ranking, or if the high class places $M H$ at the top of its ranking $-i v$ ). Finally, If $m \leq s$, then it is always the case that

$$
U_{M}^{*}\left(t^{*}\right)+m=u\left(R_{M}+T_{M}-f\left(x_{M}^{*}\left(t^{*}\right)\right)\right)<u\left(R_{A}\right)+m<u\left(R_{A}\right)+s=U^{P} .
$$

Table 1: Classes preferences over arrangements in a descending order. In Table 1a, the best separating arrangement is selected and the winning majority is made by the low and the high class. In Table $1 \mathrm{~b}$, majority preferences are cyclic.

\begin{tabular}{ccc}
$\boldsymbol{L}$ & $M$ & $\boldsymbol{H}$ \\
\hline$P$ & $M H$ & $S$ \\
$S$ & $S$ & $M H$ \\
$M H$ & $P$ & $P$
\end{tabular}

Table 1a: point iii).

\begin{tabular}{ccc}
$L$ & $M$ & $H$ \\
\hline$P$ & $M H$ & $S$ \\
$S$ & $P$ & $M H$ \\
$M H$ & $S$ & $P$
\end{tabular}

Table 1b: point $i v)$. 


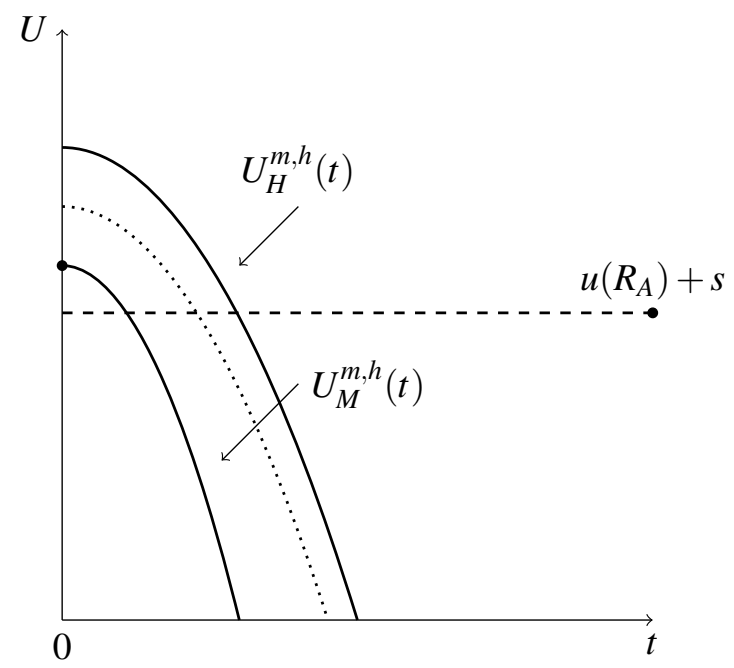

Figure 6: The parameters of the economy are $R_{L}=2, R_{M}=8, R_{H}=15$ and $l=0.05, m=0.3, h=0.8$ whereas $u(c)=\log (c)$ and $f(x)=\sqrt{x}$. Notice that $R_{M}<R_{A}$ and $m<s$. Hence, the medium class strictly prefers the best pooling to the best separating arrangement. The payoff of the medium and the high class in the $M H$ arrangement is maximized at $t^{m, h}=0$. The dotted line shows the payoff the high class would obtain in the best separating equilibrium which is lower with respect to the payoff it would obtain in the $M H$ arrangement. Therefore, the medium and the high class agree on the norm $x_{M}^{m, h}=x_{H}^{m, h}$ and the equilibrium marginal tax rate in this economy is $t^{m, h}=0$.

That is to say, if $m<s$, then the medium class always prefers the best pooling to the best separating. Nevertheless, the medium class might prefer the $M H$ arrangement to the best pooling arrangement and this arrangement could be selected if also the high class places $M H$ at the top of its ranking. ${ }^{22}$

In the extended game the average matching value in the population plays a crucial role in determining the preferences of the majority over the social arrangements and the respective tax rate. When the medium class places the $M H$ arrangement at the top of its ranking and the best pooling at its bottom, the political preferences of the medium and the lower class are zero sum. In this case, the decision is left in the hand of the high class. In turn, the high class preferences between the best separating and the $M H$ arrangement, depend on which of two effects, gain in consumption or gain in status, is larger. However, if $R_{H}$ and $h$ are sufficiently large with respect to the values of the other classes, then the loss in utility due to status consumption of the high class is negligible with respect to the gain of maintaining the matching arrangement inside the class. Nevertheless, when $m<s$ the medium class surely prefers the best pooling over the best separating arrangement. This follows from the fact that the matching payoff of the medium class is not sufficiently large to make the full separation a desired outcome. The medium class might however prefer the $M H$ arrangement inasmuch as it allows for a larger matching payoff and an increases status consumption which lowers the equilibrium marginal tax rate of redistribution. In this particular case, the social ordering over arrangements might be cyclic. Indeed, if the high class is sufficiently distant from the other classes so that she prefers the best separating, then there is no arrangement that wins any pairwise comparison. That is to say, the upward looking

${ }^{22}$ In Figure 6 I depict a society in which $m<s$ and the medium and the high class prefer the $M H$ arrangement. 
concerns of the medium class to increase its social success hinder the agreement with the low class in voting for the full redistributive arrangement. At the same time however, the best separating arrangement does not survive the comparison with the best pooling, the social ordering is cyclic and the arrangement can not be selected on the basis of classes interests. When instead the high class prefers the arrangement with the medium class, the medium class "keeps up with the Joneses" at the expense of the low class who is left in the arrangement in which economic inequality is maximized as well as her distance from the new emerging upper class $M H$. This equilibrium is depicted in Figure 6. Therefore, the last proposition underlines how a social arrangement might be grounded on a democratic and representative dimension which could deliberately exacerbate social and economic inequalities.

\section{Conclusion}

The way in which the allocation of non marketed goods takes place within a society may affect individuals preferences over different economic policies. In this article I considered a model in which the notion of social status drives matching decisions. A social arrangement is the result of a matching outcome along with a norm that prescribes how classes separate themselves signaling their relative standing by means of consuming a conspicuous good. Different norms are consistent with different matching arrangements which then shape individuals attitude towards the redistribution of wealth. There exists a natural arrangement in which classes fully separates and less then full redistribution is voted in equilibrium despite the level of economic inequality. Under some conditions however, either full redistribution of wealth occurs or the medium class pools with the high class. As a result, the desire of the medium class to reach higher success may overcome the benefits of freeing the society from economic inequality. In this case, the most conservative marginal tax rate of redistribution is voted in equilibrium along with a matching arrangement that increases the distance of the low class from the medium class. Thus, the medium class keeps up with the Joneses reaching an agreement with the high class, restricting the redistribution of wealth, at the expense of the low class. Finally, those results underline that the social organization that best represents classes interest depends on the economic conditions of the society. Therefore, changes in the economic conditions may alter classes interests and the increase of economic inequality may lead the upper classes to keep the low class away from both economic and social opportunities.

Acknowledgements Part of this work comes from my M.Sc. dissertation "Social Limits to Redistribution When People Compete for Status Through Conspicuous Consumption". I am therefore thankful to Ennio Bilancini who supervised me at the time. I am also thankful to Andrea Gallice and an anonymous referee for their precious comments. All mistakes remain mine.

\section{References}

Bilancini, E., and Boncinelli, L. (2008). Ordinal vs cardinal status: Two examples. Economics Letters, 101(1): 17-19. URL https://www.sciencedirect.com/science/article/pii/S0165176508001079.

Bilancini, E., and Boncinelli, L. (2012). Redistribution and the notion of social status. Journal of Public Economics, 96(9-10): 651-657. URL https://www.sciencedirect.com/science/article/pii/S0047272712000461. 
Bilancini, E., and Boncinelli, L. (2018). Wage inequality, labor income taxes, and the notion of social status. Economics Discussion Papers, No 2018-41, Kiel Institute for the World Economy. URL http://www.economics-ejournal.org/ economics/discussionpapers/2018-41.

Cho, I., and Kreps, D. (1987). Signaling games and stable equilibria. The Quarterly Journal of Economics, 102(2): 179-221. URL https://www.jstor.org/stable/1885060.

Cole, H. L., Mailath, G. J., and Postlewaite, A. (1992). Social norms, savings behavior, and growth. Journal of Political Economy, 100(6): 1092-1125. URL https://www.jstor.org/stable/2138828.

Corneo, G., and Gruner, H. (2000). Social limits to redistribution. American Economic Review, 90(5): 1491-1507. URL https://www.jstor.org/stable/2677862.

Duesenberry, J. (1949). Income, saving, and the theory of consumer behavior. Harvard University Press.

Frank, R. H. (1985). Choosing the right pond: Human behavior and the quest for status. Oxford University Press.

Gallice, A. (2018). Social status, preferences for redistribution and optimal taxation: A survey. Economics: The Open-Access, Open-Assessment E-Journal,, 12(2018-55): 1-17. URL http://dx.doi.org/10.5018/economics-ejournal. ja.2018-55.

Gallice, A., and Grillo, E. (2018a). Economic and social-class voting in a model of redistribution with social concerns. Carlo Alberto Notebooks, 448. URL https://ideas.repec.org/p/cca/wpaper/448.html.

Gallice, A., and Grillo, E. (2018b). A model of educational investment, social concerns, and inequality. The Scandinavian Journal of Economics, forthcoming.

Hopkins, E., and Kornienko, T. (2004). Running to keep in the same place: Consumer choice as a game of status. American Economic Review, 94(4): 1085-1107. URL https://www.aeaweb.org/articles?id=10.1257/0002828042002705.

Hopkins, E., and Kornienko, T. (2009). Status, affluence, and inequality: Rank-based comparisons in games of status. Games and Economic Behavior, 67(2): 552-568. URL https://www.sciencedirect.com/science/article/pii/ S0899825609000311.

Koenig, T., Lausen, T., and Wagener, A. (2017). Image concerns and the political economy of publicly provided private goods. Discussion paper, CESifo Working Paper No. 6304. URL https://papers.ssrn.com/sol3/papers.cfm?abstract_ $\mathrm{id}=2918724$.

Levy, G., and Razin, R. (2015). Preferences over equality in the presence of costly income sorting. American Economic Journal: Microeconomics, 7(2): 308-337. URL https://www.aeaweb.org/articles?id=10.1257/mic.20130031.

Postlewaite, A. (1998). The social basis of interdependent preferences. European Economic Review, 42(3-5): 779-800. URL http://darp.lse.ac.uk/PapersDB/Postlewaite_(EER_98).pdf.

Riley, J. G. (2001). Silver signals: Twenty-five years of screening and signaling. Journal of Economic literature, 39(2): 432-478. URL https://www.aeaweb.org/articles?id=10.1257/jel.39.2.432.

Veblen, T. (1912). The theory of the leisure class: An economic study of institutions. B.W. Huebsch.

Windsteiger, L. (2017). The redistributive consequences of segregation. Max Planck Institute for Tax Law and Public Finance Working Paper, 2017-12. URL https://papers.ssrn.com/sol3/papers.cfm?abstract_id=3091493. 


\section{Economics}

Please note:

You are most sincerely encouraged to participate in the open assessment of this article. You can do so by either recommending the article or by posting your comments.

\section{Please go to:}

http://dx.doi.org/10.5018/economics-ejournal.ja.2018-57

The Editor 